\title{
Review: herbal medicinal products seem to be effective and safe in nonulcer dyspepsia
}

Thompson Coon J, Ernst E. Systematic review: herbal medicinal products for non-ulcer dyspepsia. Aliment Pharmacol Ther 2002;16:1689-99.

\section{QUESTION: In patients with non-ulcer dyspepsia, are herbal medicinal products effective and safe?}

\section{Data sources}

Studies in any language were identified by searching Medline, EMBASE/Excerpta Medica, CINAHL, Amed, and CISCOM (all from inception to September 2001); the Cochrane Library (Issue 2, 2001); and bibliographies of relevant studies. Also, experts in the field and manufacturers of herbal products were contacted.

\section{Study selection}

Studies were selected if they were randomised controlled trials (RCTs) of herbal medicinal products given as supplements to patients with non-ulcer dyspepsia.

\section{Data extraction}

Data were extracted on study quality, sample size, patient diagnoses, intervention and control, treatment duration, outcomes, and results.

\section{Main results}

17 RCTs were included. 4 trials were of mono preparations involving greater celandine, turmeric, banana, and Emblica officinalis. In 1 RCT involving 60 patients with epigastric complaints, greater celandine reduced gastrointestinal symptoms more than did placebo at 6 weeks $(\mathrm{p}=0.003)$. In 1 RCT, patients who received turmeric $(\mathrm{n}=33), 2 \mathrm{~g} /$ day, had a higher rate of resolution or improvement in symptoms at 7 days than those who received placebo $(\mathrm{n}=20)(\mathrm{p}=0.003)$. In 1 RCT, patients who received banana powder $(\mathrm{n}=22)$ reported a greater rate of partial or complete gastrointestinal symptom relief at 8 weeks than those who received no treatment $(\mathrm{n}=24)(\mathrm{p}<0.05)$.

13 trials were of combination products, including 9 in which peppermint and caraway were constituent ingredients. In 1 RCT involving 42 patients with chronic idiopathic dyspepsia, Liu Jun Zi Tang (a combination herbal preparation), $2.5 \mathrm{~g}$ three times daily, reduced gastrointestinal symptoms more than did placebo at 7 days $(\mathrm{p}<0.05)$. In 1 RCT (100 patients), more patients who received shenxiahewining (a combination herbal preparation), $6.3 \mathrm{~g}$ /day, had symptom improvement at 20 days than those who received an unnamed control drug. In 4 RCTs of various fixed combinations of peppermint and caraway oil, treatments seemed to be effective for symptom improvement. In 1 RCT involving 60 patients with functional dyspepsia, a combination herbal preparation (peppermint leaves, caraway fruit, bitter candy tuft, licorice root, lemon balm leaves, angelica root, milk thistle fruit, and chamomile flowers) improved symptoms more than did placebo at 4 weeks $(p<0.001)$. In 1 RCT $(60$ patients), patients who received a combination of peppermint leaves, caraway fruit, fennel fruit, and wormwood herbs had less pain, nausea, and heartburn after 7 and 14 days than did those who received metaclopramide. In 1 RCT (46 patients), a combination of peppermint oil, 180 $\mathrm{mg}$ /day, and ginger extract, $25 \mathrm{mg} /$ day, improved gastrointestinal symptoms more than did placebo at 4 weeks $(p=0.012)$. Few adverse effects seemed to be associated with the herbal medicinal products, apart from reports of hepatotoxicity with greater celandine; however, comprehensive safety data are lacking.

\section{Conclusion}

In patients with non-ulcer dyspepsia, treatment with herbal medicinal products seems to be effective and safe for improving dyspeptic symptoms.

\section{COMMENTARY}

Many people have symptoms of pain or discomfort in the upper abdomen. About half of these people seek medical care, and many are investigated with endoscopy or barium radiography. Two thirds of those who are tested have no findings to explain the symptoms and are given a diagnosis of non-ulcer dyspepsia. ${ }^{1}$ The exact cause of the symptoms is not yet clear, and a clearly effective treatment has not been identified.

Given this situation, it is not surprising that many patients seek relief from alternative, non-prescription remedies. Whether such self management can be helpful is an important question. In this systematic review, Thompson Coon and Ernst summarise the literature addressing the safety and effectiveness of herbal medicinal products for the treatment of non-ulcer dyspepsia. The authors define herbal medicinal products as "any medicinal product containing as active ingredients only plants, parts of plants, or plant materials." This definition seems ambiguous: Are not many medications plant products? Is peppermint an herbal medicinal product in most people's minds? Some might consider it a food.

The authors identified 17 RCTs. Many compounds were used, and peppermint and caraway were the most common. Overall, the agents were found to be both safe and effective. However, the review highlights several important issues. Overall, the quality ratings of these trials were low. 1 study had a Jadad score of 5 (out of 5), but 9 studies scored just 1 or 2. Similar to other work in dyspepsia, the exact symptoms vary across studies. People with gastro-oesophageal reflux disease, irritable bowel syndrome, or both were neither systematically included nor excluded. Herbal preparations may have an effect on either or both of these conditions, which could confound the results. The tests used to define non-ulcer dyspepsia are also not clear. Most of the studies were small and of short duration. Another major issue is publication bias. What are the chances that a negative study of herbal preparations would be published?

Many patients improved, but that is true for almost all studies of functional gastrointestinal disorders. How do we decide that a patient with functional dyspepsia has improved? Rigorously validated symptom measures exist but have not been used in many of these studies.

Overall, this review provides evidence that more rigorous studies of these compounds are justified.

$$
\begin{array}{r}
\text { G Richard Locke III, MD } \\
\text { Mayo Clinic }
\end{array}
$$

Rochester, Minnesota, USA

1 Bytzer P, Hansen JM, Schaffalitzky de Muckadell OB. Empirical H2-blocker therapy or prompt endoscopy in management of dyspepsia. Lancet 1994;343:811-6.
Source of funding: Pharmaton SA, Switzerland.

For correspondence: Dr J Thompson Coon, Universities of Exeter and Plymouth, Exeter, $U K$. jo.thompson-coon@ pms.ac.uk

Abstract and commentary also appear in ACP Journal Club 